Ana Dorcas de Melo Inagaki

NadyeGe Pereira CARDOSO ${ }^{2}$

Renata Jule Porto Lette Lopes ${ }^{2}$

José Antônio BarReto Alves'

José Roberto Freire MESQUita ${ }^{\top}$

Karina Conceição Gomes Machado de Araújo 3 SATIE KatAGiRl

Artigo Original

Palavras-chave

Toxoplasma/imunologia Anticorpos

Complicações infecciosas na gravidez/epidemiologia Transmissão vertical de doença infeccios Estudos soroepidemiológicos

Keywords

Toxoplasma/immunology Antibodies

Pregnancy complications, infectious/epidemiology Infectious disease transmission, vertical Seroepidemiologic studies

\section{Análise espacial da prevalência de toxoplasmose em gestantes de Aracaju, Sergipe, Brasil}

\author{
Spatial distribution of anti-toxoplasma antibodies in pregnant \\ women from Aracaju, Sergipe, Brazil
}

\section{Resumo}

OBJETIVO: Analisar a distribuição espacial da prevalência de anticorpos antitoxoplasma em gestantes residentes em uma cidade do Nordeste do Brasil, e correlacionar a prevalência de anticorpos antitoxoplasma com a faixa etária materna e o local de residência. MÉTODOS: Estudo ecológico, descritivo e analítico, desenvolvido no período de 01 janeiro a 31 de dezembro de 2012. As informações foram obtidas retrospectivamente de um banco de dados, e processadas com o pacote estatístico Epi info (Epi 7, Centers for Disease Control and Prevention, Atlanta, EUA) e também em planilha do pacote Microsoft Office Excel, versão 2010. Para avaliar a associação entre a prevalência de anticorpos para a toxoplasmose e a faixa etária, foi aplicado o teste do $\chi^{2}$. A análise espacial da prevalência dessa infecção foi realizada com o programa TerraView, versão 4.2.2, utilizando o estimador de intensidade Kernel, que permite estimar a quantidade de eventos em mapa para identificar áreas de maior concentração de casos no município. RESULTADOS: A soroprevalência encontrada para lgG foi de 68,5\% (IC95\% 67,2-69,8) e lgM de 0,36\% (IC95\% 0,23-0,6). Foi encontrado incremento da prevalência de lgG associado ao aumento da idade nos bairros mais antigos da capital. Entre as mulheres mais jovens, a maior prevalência foi nos bairros de periferia. Quanto ao anticorpo IgM, a concentração espacial foi mais elevada em bairros da periferia e não ocorreu associação significativa entre a soroprevalência e a idade. CONCLUSÃO: $\bigcirc$ geoprocessamento permitiv identificar as áreas de maior prevalência, assim como a faixa etária com maior suscetibilidade, servindo como instrumento de avaliação e implementação de medidas preventivas apropriadas para esse município e outras regiões do Brasil.

\section{Abstract}

PURPOSE: To analyze the spatial distribution of the prevalence of anti-toxoplasma gondii antibodies in pregnant women from a Brazilian Northeast city, and to correlate such prevalence with average maternal age and place of residence. METHODS: A descriptive, analytical and ecological study was conducted from January $1^{\text {st }}$ to December $31^{\text {st }} 2012$. Data were obtained retrospectively from the Medical Specialties Center database and processed with the Epi info statistical package (Epi 7, Centers for Disease Control and Prevention, Atlanta, USA) and with Microsoft Excel 2010. The $\chi^{2}$ test was applied to assess the association between the prevalence of antibodies to toxoplasma gondii and the average age. Spatial analysis of infection prevalence was performed using the TerraView software, version 4.2.2, with Kernel density estimation, which estimates the quantity of events through maps in order to identify areas with the highest concentration of cases in the city. RESULTS: The seroprevalence of lgG was $68.5 \%(95 \% \mathrm{Cl} 67.2-69.8)$ and the prevalence of $\lg M$ was $0.36 \%$ 195\% Cl 0.23-0.6). A higher lgG prevalence was associated with increased age in the oldest neighborhoods of the state capital, whereas a higher lgG prevalence among younger women was detected in suburban neighborhoods. The spatial concentration of IgM antibodies was higher in suburban neighborhoods, with no significant correlation between seroprevalence and age. CONCLUSION: Geoprocessing allowed the identification of areas with the highest prevalence, as well as the most susceptible average age and it was also useful as an instrument for the evaluation and implementation of appropriate preventive measures for this municipality and for other regions of Brazil.

\section{Correspondênci \\ Ana Dorcas de Melo Inagak Rua Cláudio Batista, $s / n$ - Santo Antônio CEP: 49060-108 Aracaju (SE), Brasil \\ Recebido \\ $11 / 07 / 2014$ \\ Aceito com modificaçōes \\ $08 / 10 / 2014$}

Departamento de Enfermagem da Universidade Federal de Sergipe - UFS - Aracaju (SE), Brasil.

'Departamento de Enfermagem, Universidade Federal de Sergipe - UFS - Aracaju (SE), Brasil.

2Programa de Residência em Enfermagem Obstétrica, Universidade Federal de Sergipe - UFS - Aracaju (SE), Brasil.

${ }^{3}$ Departamento de Morfologia, Universidade Federal de Sergipe - UFS - Aracaju (SE), Brasil.

Conflito de interesses: não há. 
Introdução

A toxoplasmose assume importância significativa em gestantes devido ao risco de infecções congênitas, decorrentes da infecção aguda materna e consequente transmissão do Toxoplasma gondii por via transplacentária ${ }^{1}$. Em gestantes, essa transmissão também pode ocorrer por reinfecção ou recrudescimento ${ }^{2,3}$.

A transmissão congênita é mais frequente se a infecção for adquirida durante o terceiro trimestre de gestação, e é menor quando a infecção ocorre durante o primeiro trimestre, sendo sua gravidade inversamente proporcional. As principais sequelas para o recém-nascido são coriorretinite, hidrocefalia, calcificação cerebral ${ }^{4}$.

Em humanos, a taxa de prevalência de toxoplasmose varia entre 20 e $90 \%$ da população mundial adulta, dependendo da região, sendo mais elevada em regiões quentes e úmidas, especialmente quando associada às más condições de saneamento e hábitos alimentares ${ }^{5}$. No Brasil, a soroprevalência em gestantes varia de $31,1 \%$, em Caxias do $\mathrm{Sul}^{6}$, a 91,6\%, em Mato Grosso do Sul ${ }^{7}$. Em Sergipe, é de $69,3 \%$ e em sua capital, $77,8 \%{ }^{8}$. A soroprevalência aumenta com a idade, mas a taxa de aquisição de infecção varia de acordo com o país e o nível socioeconômico. Em populações que vivem sob precárias condições de higiene pode ser alcançada uma soroprevalência quase máxima ainda na infância? .

Sendo o pré-natal o momento favorável para educação em saúde, é importante que os profissionais de saúde conheçam os fatores de risco associados à aquisição da infecção pelo T. gondii para que ofereçam informações adequadas às gestantes sobre sua prevençãa ${ }^{10}$.

As orientações de prevenção primária devem enfatizar a importância de lavar as mãos ao manipular carne crua; evitar o consumo de carne mal cozida, água sem tratamento e leite não pasteurizado, assim como de alimentos expostos a moscas, baratas, formigas e outros insetos. Outras recomendações incluem lavar bem as frutas e legumes que serão consumidos crus. É preciso também evitar contato com gatos, utilizar luvas ao manipular fezes desses animais e o solo durante a jardinagem ou lidar com horta e agricultura ${ }^{1}$.

Uma forma atual utilizada para conhecer mais detalhadamente as condições de saúde da população é por meio da utilização de técnicas de geoprocessamento, ou seja, mediante mapas que permitem observar a distribuição espacial de situações de risco e de problemas de saúde. Essa abordagem associa informações gráficas (mapas) a bases de dados de saúde ${ }^{11}$ e permite o mapeamento das doenças contribuindo para a estruturação e análise de riscos socioambientais. O geoprocessamento proporciona, portanto, a integração de dados demográficos, socioeconômicos e de saúde ${ }^{12}$.
$\mathrm{O}$ interesse para desenvolver este estudo surgiu devido à lacuna de conhecimento quanto à distribuição espacial da infecção pelo T. gondii no Brasil. Essa análise se justifica, pois permitirá que as políticas públicas sejam direcionadas para populações com maior risco para aquisição da toxoplasmose durante a gestação e subsequente infecção congênita.

Os objetivos do presente estudo foram analisar a distribuição espacial da prevalência de anticorpos antitoxoplasma em gestantes residentes na capital do estado de Sergipe, e correlacionar a prevalência de anticorpos antitoxoplasma com a faixa etária materna e o local de residência.

\section{Métodos}

Foi desenvolvido um estudo ecológico, descritivo e analítico, realizado no período de 01 de janeiro a 31 de dezembro de 2012 em Aracaju, a capital do estado de Sergipe, Nordeste do Brasil. Aracaju está localizada na região leste do estado ( $10^{\circ} 54^{\prime} 40^{\prime \prime} \mathrm{S}$ e $\left.37^{\circ} 04^{\prime} 18^{\prime \prime} \mathrm{W}\right)$, com uma área de $181,8 \mathrm{~km}^{2}$, encontra-se a $4 \mathrm{~m}$ acima do nível do mar e tem população de 571.149 habitantes, segundo censo populacional do Instituto Brasileiro de Geografia e Estatística do ano de $2010^{13}$.

É composta por 39 bairros (Figura 1) e subdividida em 8 regiões de saúde (Figura 2). As regiões e bairros foram caracterizados de acordo com dados do Sistema de Informação da Atenção Básica (SIAB) do município do ano de 2012. A região 1 de saúde compõe a zona de expansão da cidade com bairros "praianos", como Atalaia e Coroa

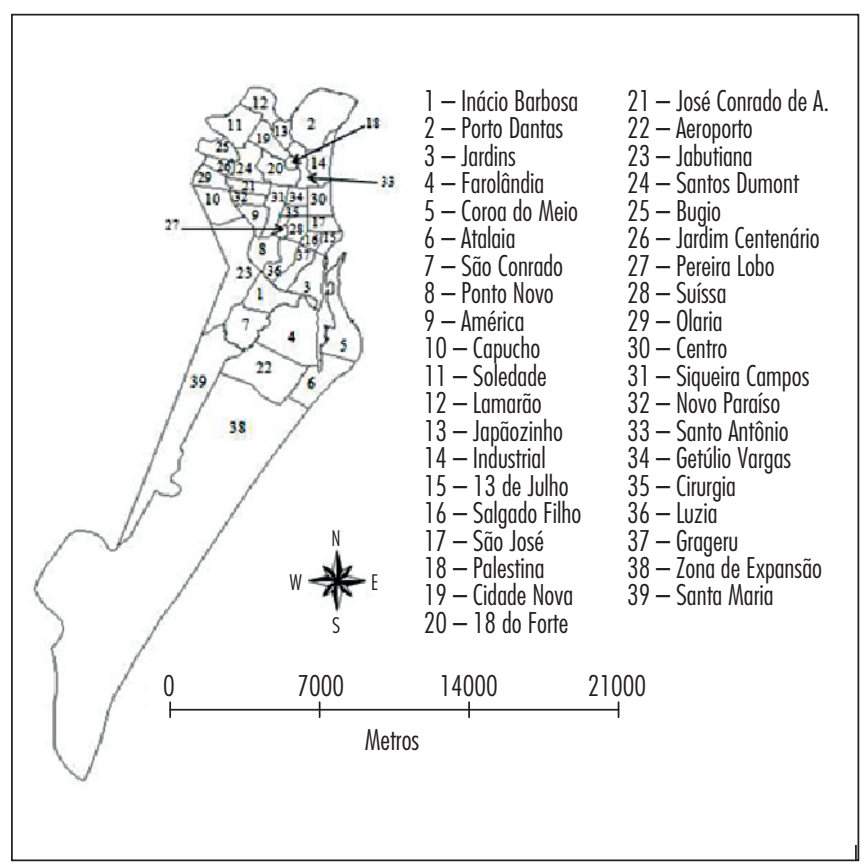

Figura 1. Mapa da cidade de Aracaju com distribuição por bairros 
do Meio, com diversas casas de veraneio, e que como a região (composta pelos bairros Jardins, Luzia e Pereira Lobo), apresenta melhores condições socioeconômicas, com uma proporção de dependentes de assistência do Sistema Único de Saúde (SUS) de 82 e 77\%, respectivamente.

Já as regiões 2, 4, 7 e 8 compreendem os bairros da periferia, como Santa Maria, José Conrado, América e Santos Dumont, habitados por famílias de menor poder socioeconômico, sendo que nas regiões 2 e 4 a dependência SUS é de $81 \%$, enquanto que nas regiões 7 e 8 é de $95 \%$.

Por fim, a região 5 compreende os bairros mais antigos da cidade, como Centro e Santo Antônio, com população na sua maioria adulta e economicamente estável e $82 \%$ de dependência SUS. A região 6, onde se localiza o bairro Suissa, a proporção de dependentes do SUS é de $92 \%$.

Os dados secundários foram coletados no Centro de Especialidades Médicas, o qual é referência para realização dos exames laboratoriais de todas as gestantes assistidas pelo SUS. A população foi constituída por gestantes residentes nesse município e a amostra foi composta por 4.883 gestantes que foram submetidas ao exame para detecção de anticorpos antitoxoplasma na ocasião da adesão ao pré-natal. O protocolo de atendimento pré-natal desse município preconiza que a pesquisa de anticorpos $\operatorname{IgM~e}$ IgG antitoxoplasma deve ser solicitada após a primeira consulta e não prevê as repetições de rotina.

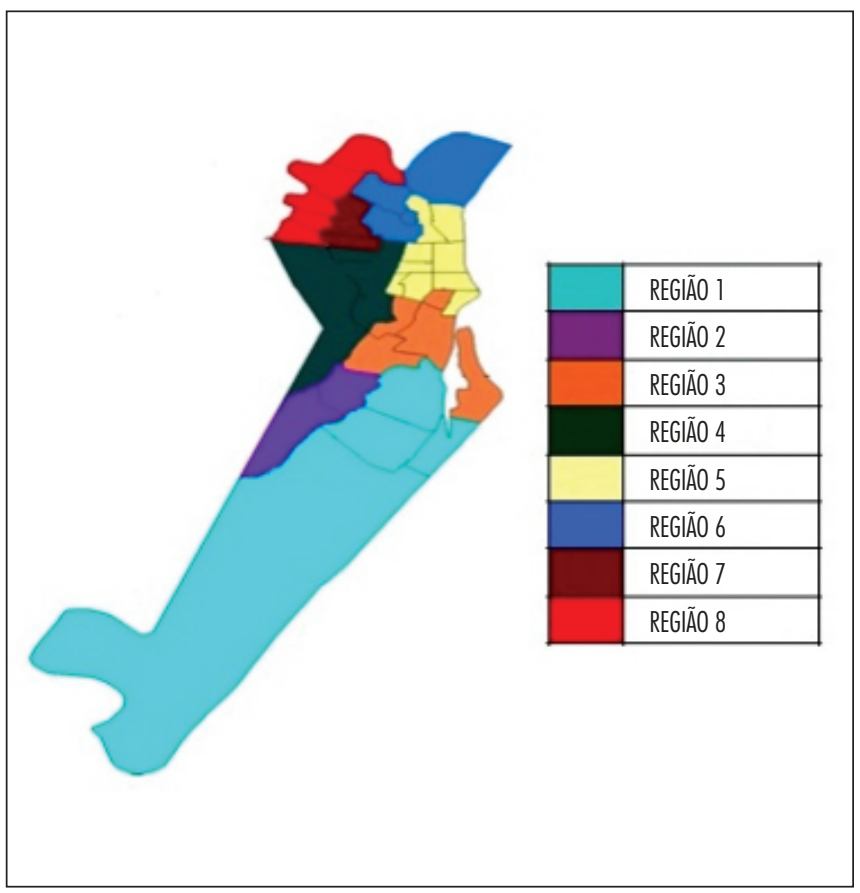

Figura 2. Mapa da cidade de Aracaju com distribuição das oito regiões de saúde. A região 1 é composta pela zona de expansão da cidade. As regiões 2, 4, 7 e 8 compreendem os bairros da periferia. A região 3 é composta por bairros nobres. As regiões 5 e 6 compreendem os bairros mais antigos da cidade
Os testes sorológicos foram realizados em amostra de sangue periférico, sendo utilizado bioelisa Toxo IgG e IgM (Biokit, S.A. Lliçà d'Amunt, Barcelona, SPAIN ${ }^{\circledR}$ ), obedecendo às instruções do fabricante.

Os dados obtidos foram processados no pacote estatístico Epi info (Epi 7, Centers for Disease Control and Prevention, Atlanta, EUA) e também em planilha do pacote Microsoft Office Excel versão 2010. Para avaliar a associação entre prevalência de anticorpos para essa infecção e a faixa etária, foi aplicado o teste do $\chi^{2}$.

A análise espacial da prevalência de toxoplasmose foi realizada no programa TerraView, versão 4.2.2, utilizando o estimador de intensidade Kernel, que permite estimar a quantidade de eventos por unidade de área em cada célula de uma grade regular que recobre a região estudada. O método de alisamento por função Kernel é uma técnica não paramétrica que promove a suavização estatística, o que permite filtrar a variabilidade de um conjunto de dados, retendo as características essenciais dos locais. Foi utilizada, na confecção dos mapas, a base cartográfica do município com eixos dos bairros. Com o objetivo de identificar áreas de maior concentração de casos no município, foram utilizados como parâmetros para gerar o mapa de Kernel, grade de 100 colunas sobre os eventos, com algoritmo de função quártica e raio adaptativo ${ }^{14}$.

Este estudo obedeceu aos princípios éticos de acordo com a Resolução 466/2012 da Comissão Nacional de Ética em Pesquisa (CONEP) e foi aprovado pelo Comitê de Ética em Pesquisa Envolvendo Seres Humanos do Hospital Universitário da Universidade Federal de Sergipe sob número CAAE 12724713.0.0000.5546.

\section{Resultados}

Foram analisados os resultados a sorologia de 4.883 gestantes, cuja idade variou entre 12 e 50 anos (média de 25,2 anos; mediana 25; e moda 24; 273 pacientes de 24 anos).

A soroprevalência encontrada para anticorpos antitoxoplasma da classe IgG foi de $68,50 \%$, com intervalo de confiança de 95\% (IC95\%) 67,2-69,8, e a da classe IgM foi de 0,36\% (IC95\% 0,2-0,6). Houve associação significativa ( $\mathrm{p}<0,001)$ entre prevalência de IgG e incremento da idade, passando de 61,30\% (IC95\% 58,5-64,0) entre as adolescentes, para 69,50\% (IC95\% 68,0-71,1) e 78,5\% (IC95\% 74,7-82,0) entre as faixa etárias de 20 a 34 anos e entre aquelas com 35 anos ou mais, respectivamente.

No que tange à possível infecção aguda, a prevalência de anticorpos antitoxoplasma da classe $\operatorname{IgM}$ foi de 0,17\% (IC95\% 0,05-0,60); 0,40\% (IC95\% 0,20-0,70) e $0,60 \%$ (IC95\% 0,20-1,70) para as faixas etárias de 10 a 19 anos, 20 a 34 anos e $\geq 35$ anos, respectivamente, não havendo diferença significante entre elas. 
A estimativa de Kernel para o número de casos de toxoplasmose na cidade, de acordo com o resultado do IgG total, IgM total e IgG por faixa etária, está apresentado na Figura 3. A gradação de cores (de verde para vermelho) quantifica a densidade de casos por bairro, ou seja, quanto mais próximo do vermelho maior a prevalência de casos nessas regiões, enquanto que o verde indica menor prevalência.

Com relação à soroprevalência de IgG por faixa etária, foi observada densidade mais elevada de ocorrência de casos na faixa etária de 10 a 19 anos em bairros localizados nas regiões de saúde 6,7 e 8 . Convém ressaltar que houve ainda prevalência relevante em um bairro da região 2 somente para essa faixa etária. Entre 20 e 34 anos ocorreu maior prevalência de IgG em bairros das regiões 3, 4 e 5 ; já entre as gestantes com idade igual ou superior a 35 anos a prevalência se concentrou somente nas regiões 3 e 4. Em relação à distribuição geral do $\mathrm{IgG}$, ocorreu maior prevalência nas regiões 3 e 5 e para o IgM nas regiões 2,4 e 7.

Quanto à distribuição das prevalências de positividade de IgG e IgM, entre as gestantes avaliadas observa-se prevalência mais elevada de anticorpo IgG nos bairros Santa Maria, Santos Dumont e São Conrado, em ordem decrescente. Situação semelhante foi encontrada quanto à ocorrência de IgM, cuja prevalência foi maior nos bairros Santa Maria e São Conrado, além do bairro América, os quais compõem as regiões de saúde 2 e 7 . Essas regiões apresentaram um percentual de $20 \%$ de gestantes adolescentes.

\section{Discussão}

A distribuição espacial tem sido utilizada na área de pesquisa em saúde por se tratar de uma análise espacial que fornece informações sobre a estrutura espacial e dinâmica da doença, caracterizando a condição de saúde em uma determinada região ${ }^{14}$.

Nossos achados indicam uma prevalência de anticorpos antitoxoplasma de 68,5\%, que está de acordo com outros estudos brasileiros ${ }^{1-15}$, resultado semelhante à pesquisa realizada em 2007 nesse mesmo município, que evidenciou soropositividade de $77,8 \%$ em $2007^{8}$. A prevalência encontrada é preocupante, considerando-se que o risco de ocorrência de toxoplasmose congênita é elevado quando a prevalência de soropositividade para toxoplasmose encontra-se entre 25 e $80 \%{ }^{16}$.

Um fato importante a ser destacado é que aproximadamente $30 \%$ das gestantes eram suscetíveis à infecção pelo T. gondii. Isso evidencia a importância de intensificar

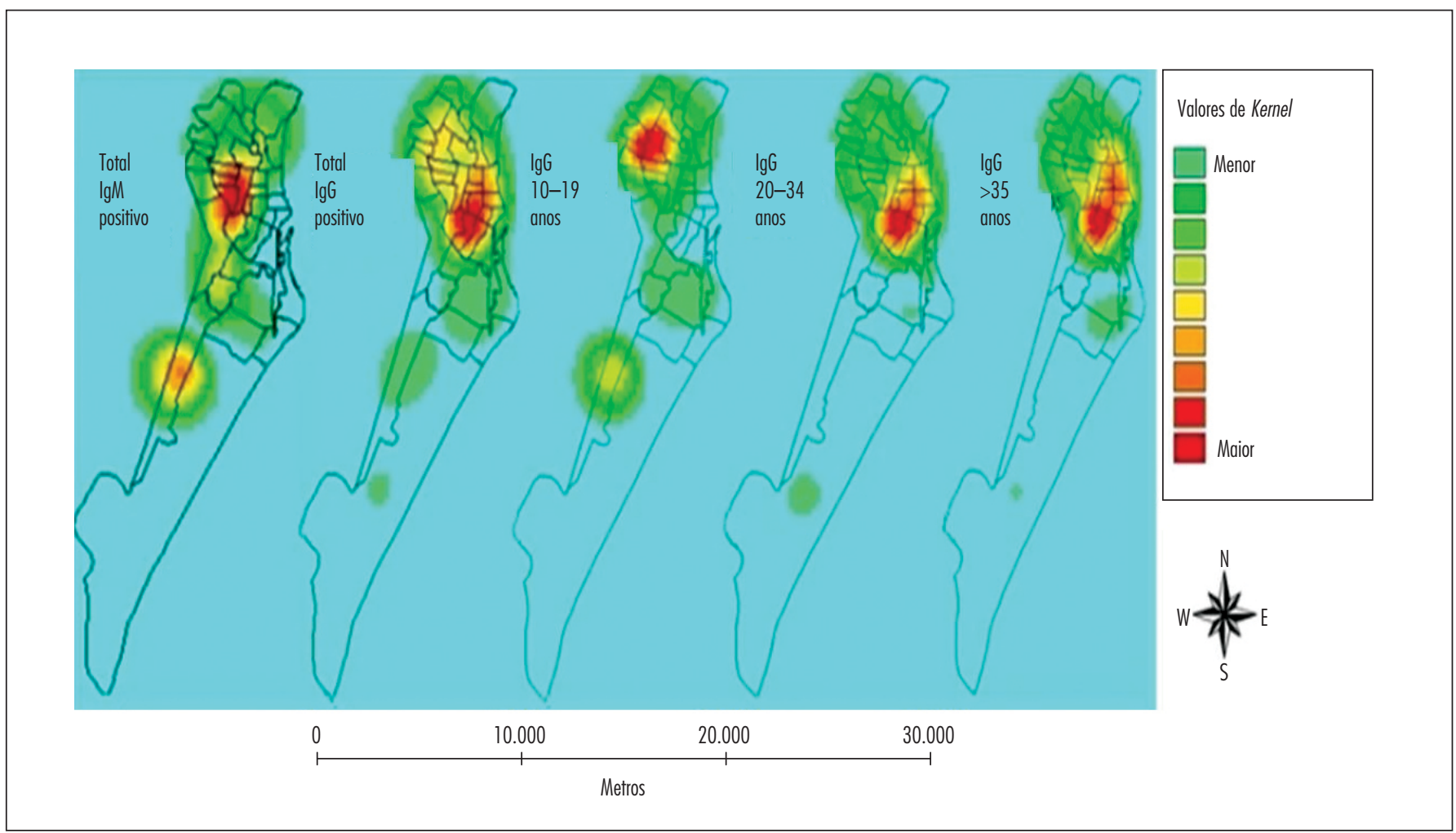

Figura 3. Distribuição espacial da toxoplasmose por variação de cores (verde para o vermelho), evidenciando o aumento da soroprevalência de anticorpos antitoxoplasma $\lg M, \lg G$ e $\lg G$ por faixa etária 
as medidas de prevenção primária devido à possibilidade de ocorrer a soroconversão durante a gestação. Estudo realizado em Goiânia, com 522 grávidas e 592 não grávidas, observou que gestantes tinham 2,2 vezes mais chance de se infectar com o parasita em relação às não grávidas ${ }^{17}$. Revelou-se ainda uma associação entre a soroprevalência de anticorpos antitoxoplasma da classe IgG e o incremento da idade das gestantes, como observado naquelas com idade igual ou superior a 35 anos, o que confirma os dados já apresentados na literatura ${ }^{8}$.

Em contrapartida, deve-se esperar que as infecções agudas com detecção de anticorpos da classe IgM ocorram mais frequentemente em pacientes mais jovens, devendo-se dar especial atenção às gestantes adolescentes, considerando que as mesmas tem risco 7,7 vezes maior de adquirir toxoplasmose ${ }^{17}$. Entretanto, nesta pesquisa não houve diferença significativa quanto a esse aspecto, situação similar à encontrada no estudo realizado na cidade do Paraná ${ }^{18}$.

É comum uma importante associação entre locais com níveis socioeconômicos baixos e o aumento da frequência de gestação em jovens ${ }^{19}$. A baixa escolaridade dessas mulheres e a consequente falta de informação sobre os cuidados preventivos para toxoplasmose a tornam propensas ao risco aumentado de infecção e subsequente transmissão ao feto ${ }^{20}$. O presente estudo confirmou essa assertiva, pois indicou soroprevalência mais elevada em gestantes jovens em bairros carentes da cidade, como Santa Maria, Lamarão e Santos Dumont, justamente os bairros com maior proporção da população dependente da assistência SUS.

No bairro Santa Maria foi desativado recentemente um aterro com todos os resíduos orgânicos recolhidos da capital, que favorecia a presença de gatos e subsequente exposição dos moradores, especialmente crianças e adolescentes. Isso pode ter contribuído para uma positividade elevada de IgG entre as gestantes mais jovens ${ }^{1}$.

Bairros com baixas condições socioeconômicas apresentaram maior proporção de infecção ativa, com IgM reagente. Entre as 18 gestantes com IgM reagente, 4 eram do bairro Santa Maria, 2 do bairro América e 2 do São Conrado, caracterizados como bolsões de pobreza. Esses dados são coerentes com os resultados de estudo realizado com 3.325 recém-nascidos no mesmo município, submetidos à triagem para toxoplasmose congênita. As duas crianças com infecção confirmada eram procedentes de bairros com as mesmas características ${ }^{21}$. Quanto à soroprevalência para IgG, observa-se uma maior densidade de gestantes com idade igual ou superior a 35 anos no Centro, São José e Suissa, bairros mais antigos da capital, o que já era esperado devido a um maior quantitativo de mulheres adultas nesses locais e melhores condições sanitárias.
A partir dos resultados apresentados observou-se que houve maior concentração de gestantes previamente infectadas pelo T. gondii residentes em áreas mais pobres, as quais estão expostas a fatores de risco conhecidos como baixo nível socioeconômico e gestação na adolescência ${ }^{17}$. Dessa forma, torna-se necessário, por parte do poder público, estabelecer estratégias direcionadas à melhoria das condições de saneamento básico, acesso à educação, incremento de ações voltadas à prevenção primária, com orientações constantes relacionadas aos hábitos higiênicos dietéticos, a fim de minimizar a exposição aos fatores de risco. Adicionalmente, há a necessidade de melhoria do programa de diagnóstico precoce da toxoplasmose, incluindo repetições da sorologia para as gestantes suscetíveis.

Embora exista um crescente interesse e aumento das pesquisas sobre a prevalência de toxoplasmose em gestantes no Brasil, ainda são raros os estudos ecológicos nessa temática, o que torna este estudo relevante. O geoprocessamento através das bases cartográficas digitais constitui apenas o ponto de partida das análises espaciais a serem georreferenciadas no campo da vigilância em saúde ${ }^{22}$.

Este estudo teve como limitação ter sido feito apenas com gestantes assistidas pelo SUS. Por se tratar de análise de banco de dados, sem contato direto com a paciente, não foi possível avaliar outras variáveis. Outra limitação foi o fato dessas gestantes não apresentarem testes confirmatórios, como o IgA e o teste de avidez para IgG. Entretanto, o grande tamanho amostral e sua representatividade com relação à população de gestantes o torna adequado para a estimativa que se pretendeu. Portanto, o presente estudo fornecerá subsídios para o georreferenciamento visando o controle e a prevenção da toxoplasmose congênita.

Em conclusão, este primeiro estudo realizado em Sergipe veio suprir uma lacuna do conhecimento de dados relevantes para o planejamento e a promoção de saúde perinatal, ao apresentar a prevalência de anticorpos antitoxoplasma de acordo com a distribuição dos casos nos diferentes bairros do município e contribuirá para a discussão acerca de medidas preventivas que são apropriadas para a realidade de cidades com características semelhantes.

\section{Agradecimentos}

Ao Centro de Especialidades Médicas, por fornecer os dados necessários para a realização deste estudo. Ao Programa de Iniciação Científica da Universidade Federal de Sergipe (UFS) pela bolsa do Programa Institucional de Bolsas de Iniciação Científica, Coordenação de Pesquisa (PIBIC-COPES). 
1. Sartori AL, Minamisava R, Avelino MM, Martins CA. [Prenatal screening for toxoplasmosis and factors associated with seropositivity of pregnant women in Goiânia, Goiás]. Rev Bras Ginecol Obstet. 2011;33(2):93-8. Portuguese.

2. Bueno WF, Ferreira RG, Silva LB, Klein CH, Amendoeira MR, Neves ES. Difficulties observed in a reference center in the diagnosis and management of pregnant women with toxoplasmosis. Sci Med. 2010;20(1):40-4.

3. Andrade GM, Vasconcelos-Santos DV, Carellos EV, Romanelli RM, Vitor RW, Carneiro AC, et al. Congenital toxoplasmosis from a chronically infected woman with reactivation of retinochoroiditis during pregnancy. J Pediatr (Rio J). 2010;86(1):85-8.

4. Zemene E, Yewhalaw D, Abera S, Belay T, Samuel A, Zeynudin A. Seroprevalence of Toxoplasma gondii and associated risk factors among pregnant women in Jimma town, Southwestern Ethiopia. BMC Infect Dis. 2012;12:337.

5. Vaz RS, Rauli P, Mello RG, Cardoso MA. Congenital toxoplasmosis: a neglected disease? - Current Brazilian public health policy. Field Act Sci Rep. 2011 ; 3:1-9.

6. Bittencourt LHFB, Lopes-Mori FMR, Mitsuka-Breganó R, ValentimZabott M, Freire RL, Pinto SB, et al. [Seroepidemiology of toxoplasmosis in pregnant women since the implementation of the Surveillance Program of Toxoplasmosis Acquired in Pregnancy and Congenital in the western region of Paraná, Brazil]. Rev Bras Ginecol Obstet. 2012;34(2):63-8. Portuguese.

7. Figueiró-Filho $E A$, Lopes $A H$, Senefonte $F R$, Souza Júnior VG, Botelho CA, Figueiredo MS, et al. [Acute toxoplasmosis: study of the frequency, vertical transmission rate and the relationship between maternal-fetal diagnostic tests during pregnancy in a Central-Western state of Brazil]. Rev Bras Ginecol Obstet. 2005;27(8):442-9. Portuguese.

8. Inagaki $A D$, Oliveira LA, Oliveira MF, Santos RC, Araújo RM, Alves JA, et al. Soroprevalência de anticorpos para toxoplasmose, rubéola, citomegalovírus, sífilis e HIV em gestantes sergipanas. Rev Soc Bras Med Trop. 2009;42(5):532-6.

9. Robert-Gangneux F, Dardé ML. Epidemiology of and diagnostic strategies for toxoplasmosis. Clin Microbiol Rev. 2012;25(2):264-96.

10. Tavares MS, Araujo RM, Abud AC, Alves JA, Nunes MS, Inagaki AD. Toxoplasmose em gestantes: prevalência, fatores de risco e ações de prevenção. Rev Enferm UFPE on line. 2012;6(6):1379-85.

11. Hau LC, Nascimento LFC, Tomazini JE. [Geoprocessing to identify the pattern of birth profile in Vale do Paraíba]. Rev Bras Ginecol Obstet. 2009;31(4):171-6. Portuguese.
12. Flauzino RF, Souza-Santos R, Oliveira RM. Dengue, geoprocessamento e indicadores socioeconômicos e ambientais: um estudo de revisão. Rev Panam Salud Publica. 2009;25(5):456-61.

13. Instituto Brasileiro de Geografia e Estatística (IBGE) [Internet]. Censo populacional. 2010 [citado 2013 Ago 02]. Disponível em: <http://www.ibge.gov.br/home/estatistica/populacao/ censo2010/default_resultados_universo.shtm>

14. Bailey TC, Gatrell AC. Interactive spatial analysis. Essex: Longman; 1995

15. Sampaio PB, Madeira ES, Diniz L, Noia EL, Zandonade E. Spatial distribution of leprosy in areas of risk in Vitória, State of Espírito Santo, Brazil, 2005 to 2009. Rev Soc Bras Med Trop. 2013;46(3):329-34.

16. Naoi K, Yano A. A theoretical analysis of the relations between the risk of congenital toxoplasmosis and the annual infection rates with a convincing argument for better public intervention. Parasitol Int. 2002;51(2): 187-94.

17. Avelino MM, Campos D Jr, Parada JC, Castro AM; Medical School of the Federal University of Goiás; Institute of Tropical Pathology and Public Health of the Federal University of Goiás; National Foundation of Support to Research; State Secretary of Health of Goiás; Municipal Secretary of Health of Goiânia. Pregnancy as a risk factor for acute toxoplasmosis seroconversion. Eur J Obstet Gynecol Reprod Biol. $2003 ; 108(1): 19-24$

18. Ferezin RI, Bertolini DA, Demarchi IG. [Prevalence of positive sorology for HIV, hepatitis B, toxoplasmosis and rubella in pregnant women from the northwestern region of the state of Paraná]. Rev Bras Ginecol Obstet. 2013;35(2):66-70. Portuguese.

19. Ferreira RA, Ferriani MG, Mello DF, Carvalho IP, Cano MA, Oliveira LA. Análise espacial da vulnerabilidade social da gravidez na adolescência. Cad Saúde Pública. 2012;28(2):313-23.

20. Costa FF, Gondim AP, Lima MB, Braga JU, Vieira L, Araújo MA. Preventive behavior for toxoplasmosis in pregnant adolescents in the state of Ceará, Brazil. BMC Public Health. $2012 ; 12: 73$.

21. Inagaki $A D$, Carvalheiro $C G$, Cipolotti R, Gurgel RQ, Rocha DA, Pinheiro KS, et al. Birth prevalence and characteristics of congenital toxoplasmosis in Sergipe, North-east Brazil. Trop Med Int Health. 2012;17(11):1349-55.

22. Garnelo L, Brandão LC, Levino A. Dimensões e potencialidades dos sistemas de informação geográfica na saúde indígena. Rev Saúde Pública. 2005;39(4):634-40. 